\title{
Modified Slash Lindley Distribution
}

\author{
Jimmy Reyes, ${ }^{1}$ Osvaldo Venegas, ${ }^{2}$ and Héctor W. Gómez ${ }^{1}$ \\ ${ }^{1}$ Departamento de Matemáticas, Facultad de Ciencias Básicas, Universidad de Antofagasta, Antofagasta, Chile \\ ${ }^{2}$ Departamento de Ciencias Matemáticas y Físicas, Facultad de Ingeniería, Universidad Católica de Temuco, Temuco, Chile
}

Correspondence should be addressed to Osvaldo Venegas; ovenegas@uctemuco.cl

Received 27 September 2016; Revised 17 January 2017; Accepted 29 January 2017; Published 19 February 2017

Academic Editor: Ramón M. Rodríguez-Dagnino

Copyright (C) 2017 Jimmy Reyes et al. This is an open access article distributed under the Creative Commons Attribution License, which permits unrestricted use, distribution, and reproduction in any medium, provided the original work is properly cited.

\begin{abstract}
In this paper we introduce a new distribution, called the modified slash Lindley distribution, which can be seen as an extension of the Lindley distribution. We show that this new distribution provides more flexibility in terms of kurtosis and skewness than the Lindley distribution. We derive moments and some basic properties for the new distribution. Moment estimators and maximum likelihood estimators are calculated using numerical procedures. We carry out a simulation study for the maximum likelihood estimators. A fit of the proposed model indicates good performance when compared with other less flexible models.
\end{abstract}

\section{Introduction}

The Lindley distribution was introduced by Lindley [1] with the density function given by

$$
f_{X}(x)=\frac{\lambda^{2}}{1+\lambda}(1+x) e^{-\lambda x}, \quad \lambda>0, x>0 .
$$

We denote this by writing $X \sim L(\lambda)$, where $\lambda$ is the shape parameter. The corresponding cumulative distribution function (c.d.f.) is

$$
F_{X}(x)=1-\frac{1+\lambda+\lambda x}{1+\lambda} e^{-\lambda x} .
$$

The properties of the Lindley distribution are studied in detail by Ghitany et al. [2]. Jodrá [3] uses the Lambert $W$ function for the generation of random variables with Lindley or Poisson-Lindley distributions. Ghitany et al. [4] propose the power Lindley (PL) distribution generated from a random variable $X \sim L(\lambda)$ raised to the power $1 / \alpha$; that is, if $Y=X^{1 / \alpha}$ then $Y$ has PL distribution of parameters $\alpha$ and $\lambda$, with density function given by

$$
f_{Y}(y)=\frac{\alpha \lambda^{2}}{1+\lambda}\left(1+y^{\alpha}\right) e^{-\lambda y^{\alpha}}, \quad \alpha>0, \lambda>0, \quad y>0
$$

We denote this by writing $Y \sim \operatorname{PL}(\alpha, \lambda)$. The half-normal distribution is suitable for fitting positive data. We say that a random variable $X$ follows a half-normal distribution with scale parameter $\sigma$ if its density function is given by

$$
f_{X}(x)=\frac{2}{\sigma} \phi\left(\frac{x}{\sigma}\right), \quad \sigma>0, x>0
$$

where $\phi(\cdot)$ represents the density function of the standard normal distribution; we denote this by writing $X \sim \mathrm{HN}(\sigma)$.

Olmos et al. [5] introduce a new distribution suitable for fitting positive data called the slash half-normal distribution, which is a distribution with right-tails heavier than the $\mathrm{HN}$ distribution. It is obtained as a particular case when the shape parameter $q$ tends to infinity. We say that a random variable $X$ follows a slash half-normal (SHN) distribution with scale parameter $\sigma$ and kurtosis parameter $q$ if its density function is given by

$$
f_{X}(x)=\frac{2 q}{\sigma} \int_{0}^{1} u^{q} \phi\left(\frac{x u}{\sigma}\right) d u, \quad \sigma>0, q>0, x>0 .
$$

We denote this by writing $X \sim \operatorname{SHN}(\sigma, q)$.

In the study of symmetric distributions with heavy tails, Reyes et al. [6] introduce a modification of the class of standard slash distributions, which will be called modified 
slash (MS) distribution and is described as follows: We will say that $X$ has MS distribution with parameter $q>0$ if it can be expressed as

$$
X=\frac{Z}{U^{1 / q}}
$$

where $Z \sim N(0,1)$ and $U \sim \operatorname{Exp}(2)$ for $q>0$. Here $Z$ and $U$ are independent random variables, denoted by $X \sim \operatorname{MS}(0,1, q)$; the density function of $X$ has heavier tails than the standard slash distribution and in consequence has higher kurtosis. When $q \rightarrow \infty$ we obtain the standard normal distribution. The density function of the variable $X \sim$ $\operatorname{MS}(0,1, q)$ is given by

$$
\begin{aligned}
f_{X}(x)=\frac{2}{\sqrt{2 \pi}} \int_{0}^{\infty} v^{1 / q} e^{-(1 / 2) x^{2} v^{2 / q}-2 v} d v & \\
& \\
& q>0, x \in \mathbb{R}
\end{aligned}
$$

where $q$ is kurtosis parameter; see Reyes et al. [6] for more details. Using the same idea, Reyes et al. [7] extend the skewnormal model and Reyes et al. [8] extend the BirnbaumSaunders model. Gui [9] introduces the slash Lindley (SL) distribution and applies it to data on precipitation and plasma ferritin concentration. Gui et al. [10] introduce the LindleyPoisson (LP) distribution and apply it to lifetime data.

The focus of this paper is the introduction of a new distribution called modified slash Lindley (MSL) distribution. Because of its mixed approach, the newly constructed distribution will have heavier tails than its parent Lindley distribution and hence will be more suitable for modeling positive data sets that may have heavy tails and/or outliers. This new distribution is quite appropriate for modelling positive data with very atypical observations (outliers); as we can see in the application, the MSL distribution MSL better models data on the survival cancer patients with atypical remission times.

The paper is organized as follows. Section 2 is devoted to the development of a stochastic representation for the MSL distribution and its use for density function derivation and also the derivation of its moments, asymmetry and kurtosis coefficients. In Section 3, the inference is discussed for the MSL distribution using the method of moments estimators and maximum likelihood estimation. We also present an illustrative example with real data on survival times. This example shows that the proposed distribution is a very appropriate model for this data set.

\section{MSL Distribution}

In this section we consider a stochastic representation, the density function (with some graphical representations), and properties of the modified slash Lindley distribution.

2.1. Stochastic Representation. The stochastic representation of the new distribution is given as

$$
Y=\frac{X}{U^{1 / q}}
$$

where $X \sim L(\lambda)$ and $U \sim \operatorname{Exp}(2)$ are independent random variables with $\lambda>0, q>0$. We called the distribution of $Y$ the MSL distribution, and we use the notation $Y \sim \operatorname{MSL}(\lambda, q)$.

2.2. Density Function. The following result shows that the density function of the random variable MSL can be generated using the stochastic representation in (8).

Proposition 1. Let $Y \sim \operatorname{MSL}(\lambda, q)$. Then, the density function of $Y$ is given by

$$
\begin{array}{r}
f_{Y}(y)=\frac{2 \lambda^{2}}{1+\lambda} \int_{0}^{\infty} w^{1 / q}\left(1+y w^{1 / q}\right) e^{-\left(\lambda y w^{1 / q}+2 w\right)} d w \\
y>0
\end{array}
$$

with $\lambda>0$ and $q>0$.

Proof. Using the stochastic representation in (8) and from the Jacobian transformation approach it follows that

$$
\begin{aligned}
Y & =\frac{X}{U^{1 / q}} \\
W & =U \\
\Downarrow & \\
X & =Y W^{1 / q} \\
U & =W \\
\Downarrow & \\
J & =\left|\begin{array}{ll}
\frac{\partial X}{\partial Y} & \frac{\partial X}{\partial W} \\
\frac{\partial U}{\partial Y} & \frac{\partial U}{\partial W}
\end{array}\right|=\left|\begin{array}{cc}
w^{1 / q} & \left(\frac{1}{q}\right) y w^{(1 / q)-1} \\
0 & 1
\end{array}\right|=w^{1 / q} .
\end{aligned}
$$

Hence,

$$
\begin{aligned}
& f_{Y, W}(y, w)=|J| f_{X, U}\left(y w^{1 / q}, w\right) \\
&=w^{1 / q} f_{X}\left(y w^{1 / q}\right) f_{U}(w) \\
&=\frac{\lambda^{2}}{1+\lambda} w^{1 / q}\left(1+y w^{1 / q}\right) e^{-\lambda y w^{1 / q}} 2 e^{-2 w}, \\
& y>0, w>0 .
\end{aligned}
$$

By marginalizing, the result follows immediately.

The following proposition shows that the MSL distribution results from a mixture of the LI distribution on the scale parameter $w^{-1 / q}$ and shape parameter $\lambda$, and the exponential distribution with parameter equals two.

Proposition 2. Let $Y \mid W=w \sim L\left(w^{-1 / q}, \lambda\right)$ and $W \sim$ $\operatorname{Exp}(2)$; then $Y \sim \operatorname{MSL}(\lambda, q)$. 


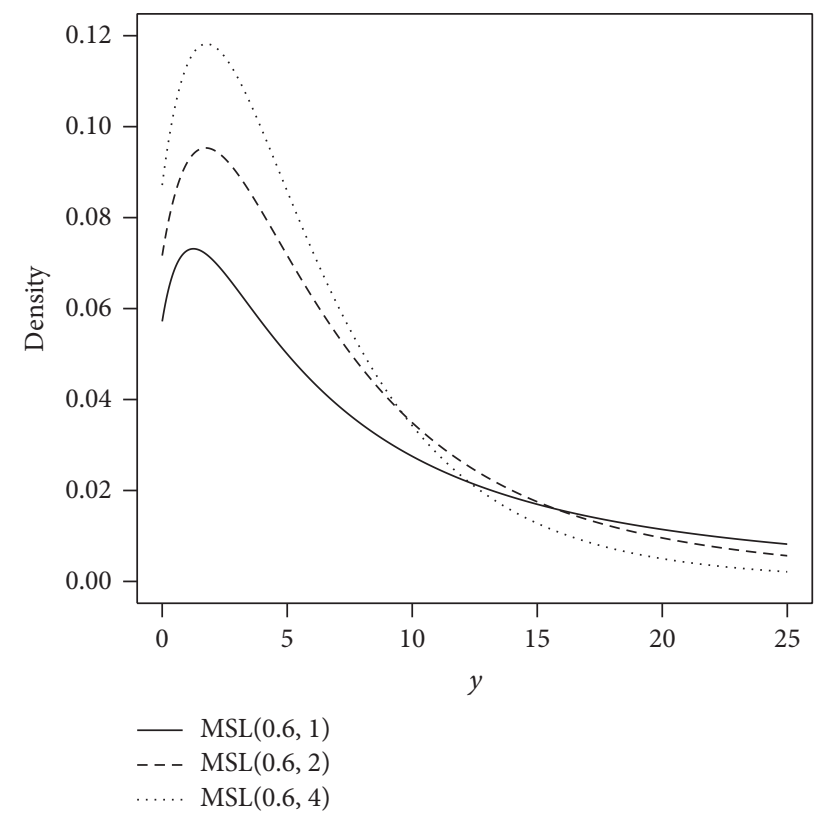

(a)

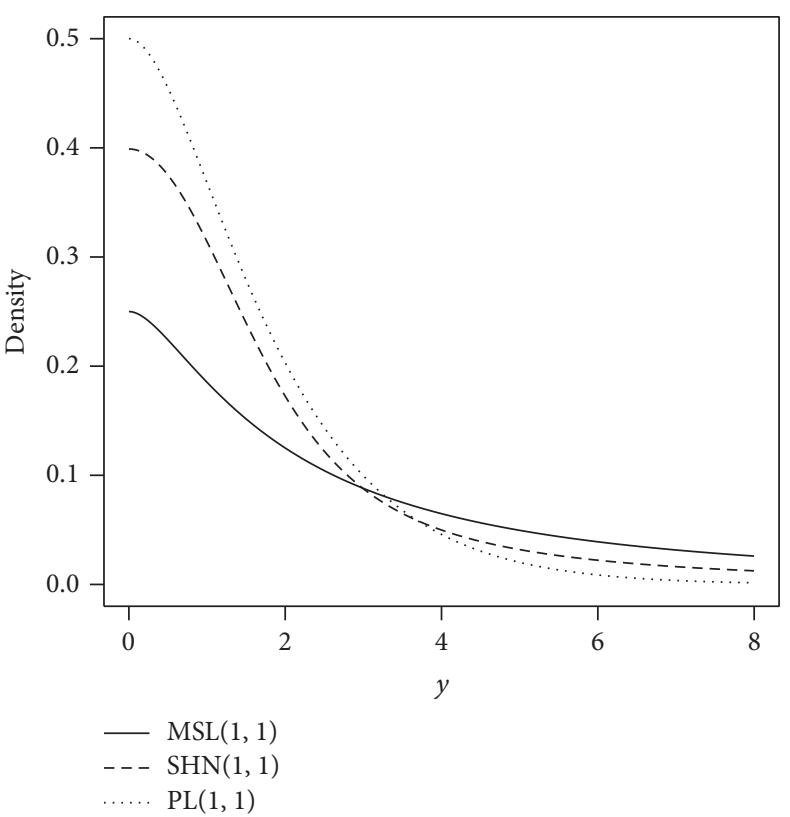

(b)

FIGURE 1: Density functions for the MSL distributions with $\lambda=0.6$ and several values of $q$ (a), compared with SHN and PL distribution (b).

Proof. We can write

$$
\begin{aligned}
& f_{Y}(y) \\
& =\int_{0}^{\infty} \frac{\lambda^{2}}{(1+\lambda) w^{-1 / q}}\left(1+\frac{y}{w^{-1 / q}}\right) e^{-\lambda y / w^{-1 / q}} 2 e^{-2 w} d w \\
& =\frac{2 \lambda^{2}}{1+\lambda} \int_{0}^{\infty} w^{1 / q}\left(1+w^{1 / q} y\right) e^{-\left(\lambda w^{1 / q} y+2 w\right)} d w .
\end{aligned}
$$

In Figure 1, we illustrate the behavior of the density function of the MSL distribution.

\subsection{Moments}

Proposition 3. If $Y \sim \operatorname{MSL}(\lambda, q)$, the rth moment of $Y$ is given by

$$
\mu_{r}=E\left[Y^{r}\right]=\frac{r !(\lambda+r+1)}{\lambda^{r}(\lambda+1)}\left[2^{r / q} \Gamma\left(\frac{q-r}{q}\right)\right],
$$

$$
q>r \text {. }
$$

Proof. From the stochastic representation given in (8), in which $X \sim L(\lambda)$ and $U \sim \operatorname{Exp}(2)$ are independent random variables, we have

$$
E\left[Y^{r}\right]=E\left[\left(\frac{X}{U^{1 / q}}\right)^{r}\right]=E\left[X^{r}\right] E\left[U^{-r / q}\right]
$$

from which both expectations are known.

Remark 4. In Figure 1 it is observed that when parameter $\lambda$ is less than 1, the distribution is unimodal; see Figure 1(a).

Corollary 5. Let $Y \sim \operatorname{MSL}(\lambda, q)$. Then it follows that

$$
\begin{aligned}
& \mu_{1}=E(Y)=\frac{(\lambda+2)}{\lambda(\lambda+1)}\left[2^{1 / q} \Gamma\left(\frac{q-1}{q}\right)\right], \quad q>1, \\
& \mu_{2}=E\left(Y^{2}\right)=\frac{2(\lambda+3)}{\lambda^{2}(\lambda+1)}\left[2^{2 / q} \Gamma\left(\frac{q-2}{q}\right)\right], \quad q>2, \\
& \mu_{3}=E\left(Y^{3}\right)=\frac{6(\lambda+4)}{\lambda^{3}(\lambda+1)}\left[2^{3 / q} \Gamma\left(\frac{q-3}{q}\right)\right], \quad q>3, \\
& \mu_{4}=E\left(Y^{4}\right)=\frac{24(\lambda+5)}{\lambda^{4}(\lambda+1)}\left[2^{4 / q} \Gamma\left(\frac{q-4}{q}\right)\right], \quad q>4 .
\end{aligned}
$$

Proof. It is a direct consequence of Proposition 3.

Corollary 6. Let $Y \sim \operatorname{MSL}(\lambda, q)$. Then the skewness coefficient is

$$
\gamma_{1}=\frac{6(\lambda+4)(\lambda+1)^{2} \kappa_{3}-6(\lambda+1)(\lambda+2)(\lambda+3) \kappa_{1} \kappa_{2}+2(\lambda+2)^{3} \kappa_{1}^{3}}{\left[2(\lambda+1)(\lambda+3) \kappa_{2}-(\lambda+2)^{2} \kappa_{1}^{2}\right]^{3 / 2}}
$$




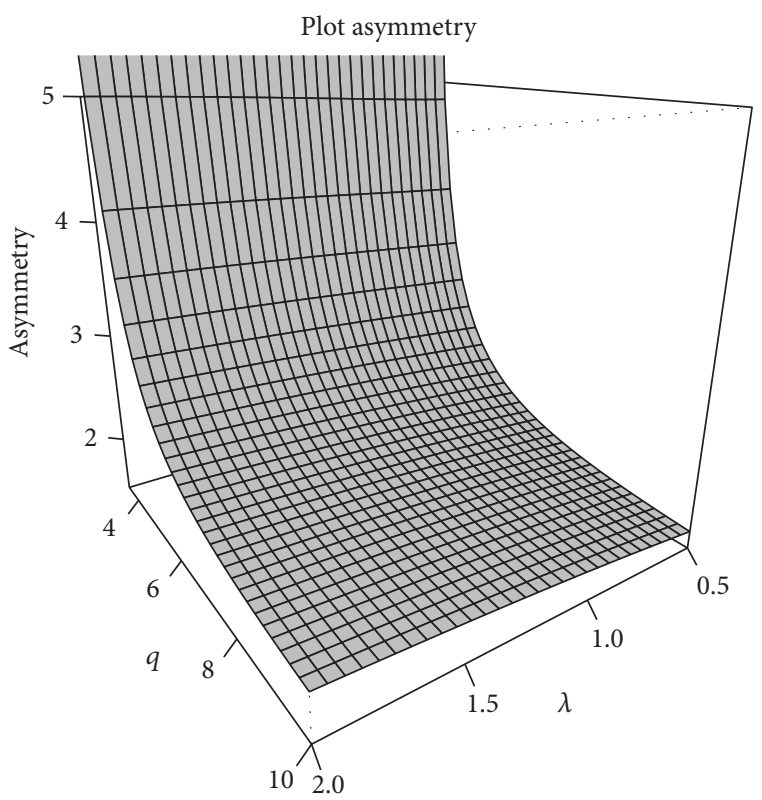

(a)

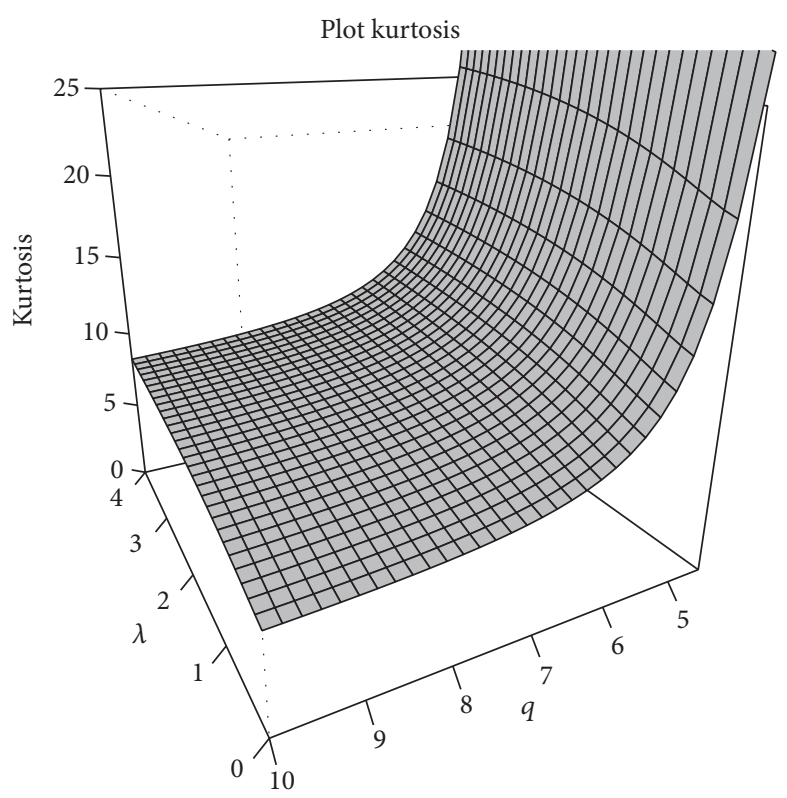

(b)

FIGURE 2: Asymmetry (a) and kurtosis (b) for several values of $\lambda$ and $q$ for the MSL distributions.

and the kurtosis coefficient is

$$
\begin{aligned}
\gamma_{2} & =\frac{24(\lambda+1)^{3}(\lambda+5) \kappa_{4}+12(\lambda+1)(\lambda+2) \kappa_{1}\left[(\lambda+2)(\lambda+3) \kappa_{1} \kappa_{2}-2(\lambda+4)(\lambda+1) \kappa_{3}\right]}{\left[2(\lambda+1)(\lambda+3) \kappa_{2}-(\lambda+2)^{2} \kappa_{1}^{2}\right]^{2}} \\
& -\frac{3(\lambda+2)^{4} \kappa_{1}^{4}}{\left[2(\lambda+1)(\lambda+3) \kappa_{2}-(\lambda+2)^{2} \kappa_{1}^{2}\right]^{2}},
\end{aligned}
$$

where $\kappa_{i}=\Gamma((q-i) / q)$, with $i=1,2,3,4$.

Proof. The result is obtained by using the following skewness and kurtosis coefficients

$$
\begin{aligned}
& \gamma_{1}=\frac{\mathbb{E}[Y-\mathbb{E}(Y)]^{3}}{[\operatorname{Var}(Y)]^{3 / 2}}=\frac{\mu_{3}-3 \mu_{2} \mu_{1}+2 \mu_{1}^{3}}{\left(\mu_{2}-\mu_{1}^{2}\right)^{3 / 2}}, \\
& \gamma_{2}=\frac{\mathbb{E}[Y-\mathbb{E}(Y)]^{4}}{[\operatorname{Var}(Y)]^{2}}=\frac{\mu_{4}-4 \mu_{1} \mu_{3}+6 \mu_{1}^{2} \mu_{2}-3 \mu_{1}^{4}}{\left(\mu_{2}-\mu_{1}^{2}\right)^{2}} .
\end{aligned}
$$

In Figure 2, we illustrate the behavior of the asymmetry and kurtosis of the MSL distribution as a function of $\lambda$ and $q$.

Remark 7. It can be seen that for small values of the parameter $q$ the asymmetry and kurtosis coefficients are high. Also, for high values of the parameter $q$ the asymmetry and kurtosis coefficients tend to the coefficients of the $L$ distribution.

\section{Inference}

In this section, we study the parameter estimation of the new model using the maximum likelihood and moments approach.

3.1. Method of Moments Estimation. Let $Y_{1}, Y_{2}, \ldots, Y_{n}$ be a random sample from the $\operatorname{MSL}(\lambda, q)$ distribution that has density function given by (9). Then using (15) and (16) and replacing $\bar{y}$ by $E(Y)$ and $\overline{y^{2}}$ by $E\left(Y^{2}\right)$ we have the following system:

$$
\begin{aligned}
& \bar{y}=\frac{(\lambda+2)}{\lambda(\lambda+1)}\left[2^{1 / q} \Gamma\left(\frac{q-1}{q}\right)\right], \\
& \overline{y^{2}}=\frac{2(\lambda+3)}{\lambda^{2}(\lambda+1)}\left[2^{2 / q} \Gamma\left(\frac{q-2}{q}\right)\right] .
\end{aligned}
$$

Using an appropriate numerical method we solve the equation system for $\lambda$ and $q$. Hence we obtain the moment estimators $\widehat{\boldsymbol{\theta}}_{M}=\left(\widehat{\lambda}_{M}, \widehat{q}_{M}\right)$ of $\boldsymbol{\theta}=(\lambda, q)$. 
In the following proposition the asymptotic convergence of these estimators is presented.

Proposition 8. Let $Y_{1}, Y_{2}, \ldots, Y_{n}$ be a random sample from the $\operatorname{MSL}(\lambda, q)$ distribution. Let $\boldsymbol{\theta}=(\lambda, q)$ and for $k=1,2,3,4$ denote $\mu_{k}(\boldsymbol{\theta})=E\left(Y^{k}\right)$. If $\mu_{4}<\infty$ and $\widehat{\boldsymbol{\theta}}_{M}(n)$ are corresponding moments estimators, we have

$$
\sqrt{n}\left(\widehat{\boldsymbol{\theta}}_{M}(n)-\boldsymbol{\theta}\right) \stackrel{\mathscr{D}}{\longrightarrow} N_{2}(0, \Gamma(\boldsymbol{\theta})), \quad n \longrightarrow+\infty,
$$

where

$$
\begin{aligned}
\Gamma(\boldsymbol{\theta}) & =H^{-1}(\boldsymbol{\theta}) \sum\left[H^{-1}(\boldsymbol{\theta})\right]^{T}, \\
\sum & =\left\{\left(\mu_{i+j}-\mu_{i} \mu_{j}\right)_{i j}\right\}, \\
H(\boldsymbol{\theta}) & =\left(\begin{array}{cc}
-\left(\frac{\lambda^{2}+4 \lambda+2}{\lambda^{2}(\lambda+1)^{2}}\right) \tau_{1} & \frac{(\lambda+2) \tau_{1}}{\lambda(\lambda+1) q^{2}}\left[\psi\left(\frac{q-1}{q}\right)-\log (2)\right] \\
-\left(\frac{4 \lambda^{2}+20 \lambda+12}{\lambda^{3}(\lambda+1)^{2}}\right) \tau_{2} & \frac{4(\lambda+3) \tau_{2}}{\lambda^{2}(\lambda+1) q^{2}}\left[\psi\left(\frac{q-2}{q}\right)-\log (2)\right]
\end{array}\right),
\end{aligned}
$$

where $A(\boldsymbol{\theta})=\left(\mu_{1}(\boldsymbol{\theta}), \mu_{2}(\boldsymbol{\theta})\right), H(\boldsymbol{\theta})=\partial A(\boldsymbol{\theta}) / \partial(\boldsymbol{\theta})$, and $\psi(\cdot)$ is the function digamma and $\tau_{i}=2^{i / q} \kappa_{i}$, with $i=1,2$.

Proof. It follows directly by using standard large sample theory results for moment estimators, as discussed for example, in Sen and Singer [11].

3.2. Maximum Likelihood Estimation. We will now discuss maximum likelihood estimation. Given a random sample $Y_{1}, \ldots, Y_{n}$ from the distribution of $\operatorname{MSL}(\lambda, q)$, the log likelihood function can be written as

$$
\begin{aligned}
\ell(\lambda, q)= & n \log (2)+2 n \log (\lambda)-n \log (\lambda+1) \\
& +\sum_{i=1}^{n} \log \left[G\left(y_{i}\right)\right]
\end{aligned}
$$

where $G\left(y_{i}\right)=\int_{0}^{\infty} u^{1 / q}\left(1+y_{i} u^{1 / q}\right) e^{-\left(\lambda y_{i} u^{1 / q}+2 u\right)} d u$ and hence the maximum likelihood equation is given by

$$
\begin{aligned}
& \sum_{i=1}^{n} \frac{G_{1}\left(y_{i}\right)}{G\left(y_{i}\right)}=-\frac{2 n}{\lambda}+\frac{n}{\lambda+1}, \\
& \sum_{i=1}^{n} \frac{G_{2}\left(y_{i}\right)}{G\left(y_{i}\right)}=0,
\end{aligned}
$$

where the expressions for $G_{1}\left(y_{i}\right)$ and $G_{2}\left(y_{i}\right)$ should be given

$$
\begin{gathered}
G_{1}\left(y_{i}\right)=\frac{\partial}{\partial \lambda} G\left(y_{i}\right)=-\int_{0}^{\infty} u^{1 / q} w_{i}\left(1+w_{i}\right) \\
\cdot e^{-\left(\lambda w_{i}+2 u\right)} d u, \\
G_{2}\left(y_{i}\right)=\frac{\partial}{\partial q} G\left(y_{i}\right)=\frac{1}{q^{2}} \int_{0}^{\infty} u^{1 / q} \log (u) \\
\cdot e^{-\left(\lambda w_{i}+2 u\right)}\left[(\lambda-2) w_{i}+\lambda w_{i}^{2}-1\right] d u,
\end{gathered}
$$

where $w_{i}=y_{i} u^{1 / q}$.
Solutions for (26) can be obtained using numerical procedures such as the Newton-Raphson procedure.

3.2.1. Simulation Study. By using the representation considered by Ghitany et al. [2] to generate random numbers of a random variable $L$ and the representation given in (8), it is possible to generate random numbers for the $\operatorname{MSL}(\lambda, q)$ distribution, leading to the following algorithm:

(1) Simulate $S \sim \operatorname{Exp}(\lambda)$.

(2) Simulate $T \sim \operatorname{Gamma}(2, \lambda)$.

(3) Simulate $V \sim \operatorname{Bernulli}(\lambda /(1+\lambda))$.

(4) Compute $X=V S+(1-V) T$.

(5) Simulate $U \sim \operatorname{Exp}(2)$.

(6) Compute $Y=X / U^{1 / q}$.

It then follows that $Y \sim \operatorname{MSL}(\lambda, q)$.

Table 1 shows the results of simulations studies illustrating the behavior of the MLEs for 1000 generated samples of sizes 50, 100, 150, and 200 from population distributed as $\operatorname{MSL}(\lambda, q)$. For each sample generated, MLEs were computed numerically using a Newton-Raphson procedure. Means and standard deviations (SD) are reported. It is observed that the bias becomes smaller as the sample size $n$ increases, as one would expect.

3.3. An Illustrative Example with a Real Data Set. We consider here a data set representing the remission times (in months) of a random sample of 128 bladder cancer patients as reported in Lee and Wang [12]. The data are as follows: 0.08, 2.09, $3.48,4.87,6.94,8.66,13.11,23.63,0.20,2.23,3.52,4.98,6.97$, $9.02,13.29,0.40,2.26,3.57,5.06,7.09,9.22,13.80,25.74,0.50$, $2.46,3.64,5.09,7.26,9.47,14.24,25.82,0.51,2.54,3.70,5.17$, $7.28,9.74,14.76,26.31,0.81,2.62,3.82,5.32,7.32,10.06,14.77$, $32.15,2.64,3.88,5.32,7.39,10.34,14.83,34.26,0.90,2.69,4.18$, $5.34,7.59,10.66,15.96,36.66,1.05,2.69,4.23,5.41,7.62,10.75$, 


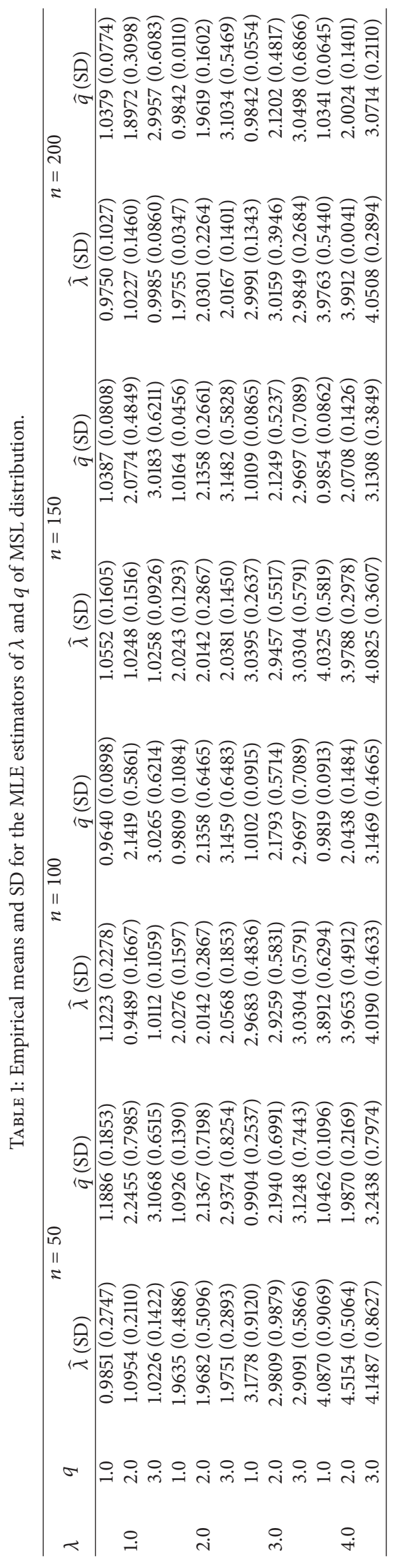



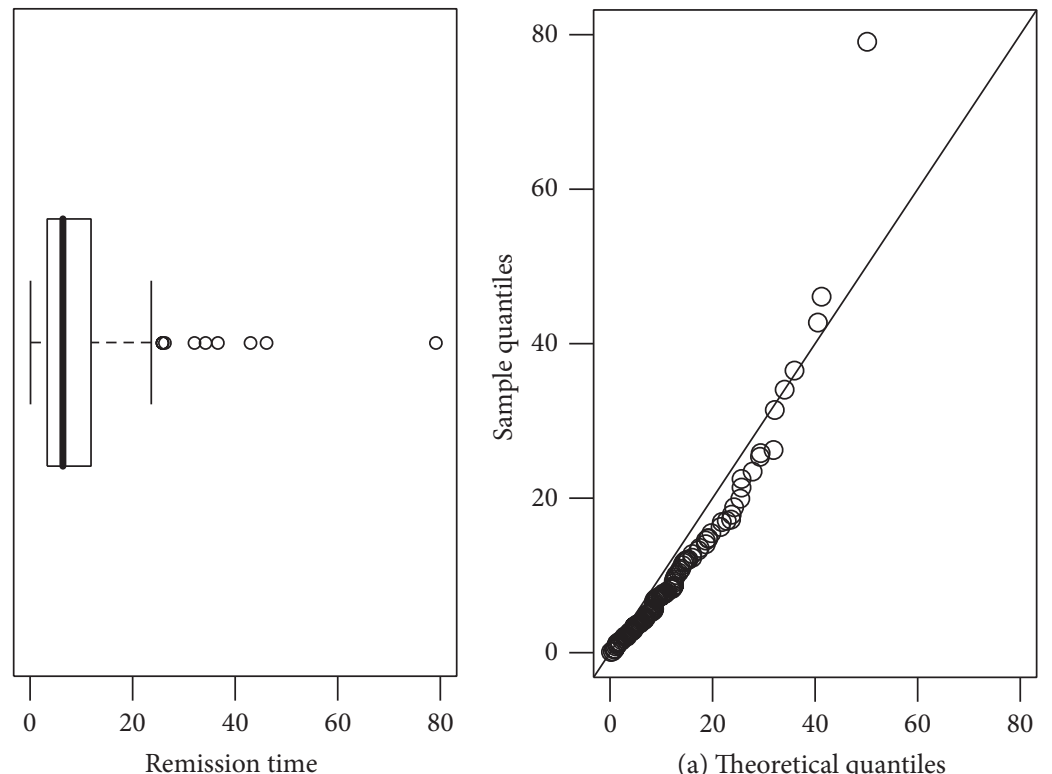

(a) Theoretical quantiles

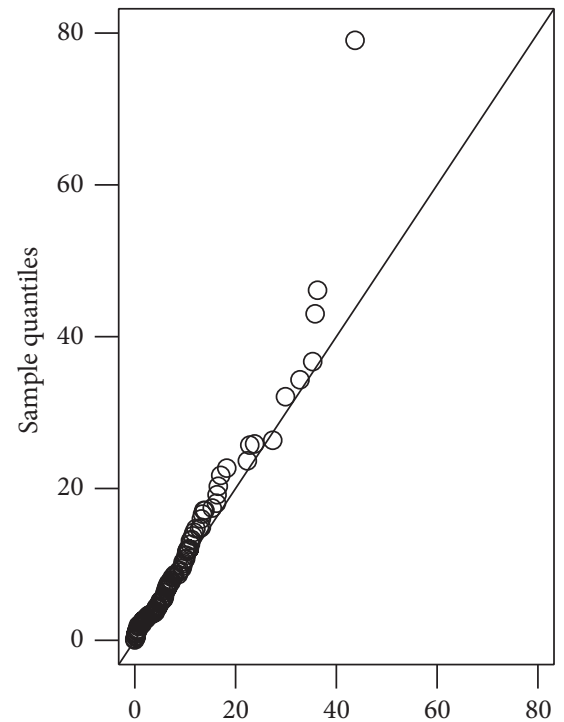

(c) Theoretical quantiles

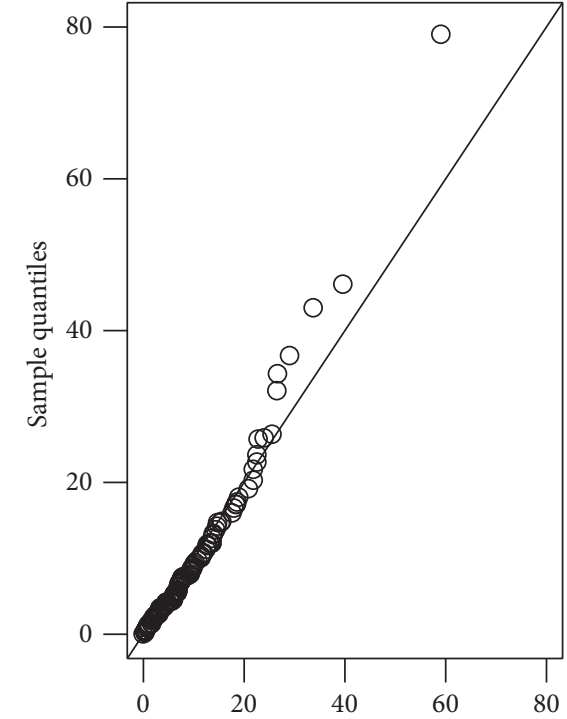

(d) Theoretical quantiles

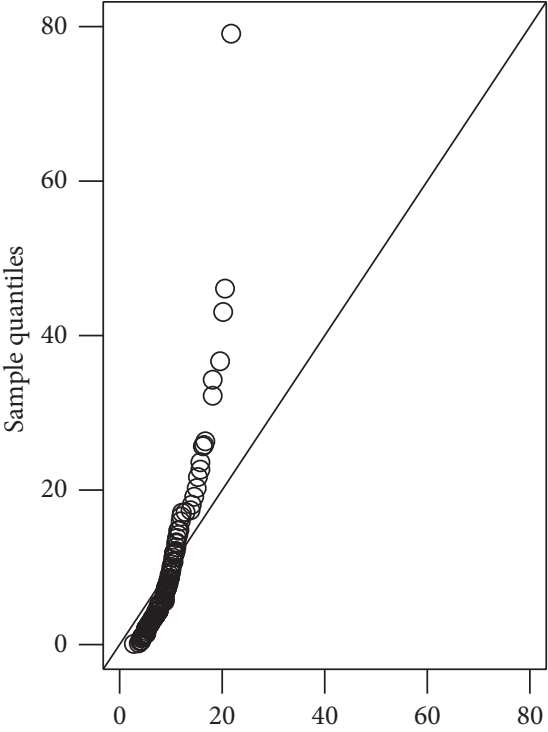

(b) Theoretical quantiles

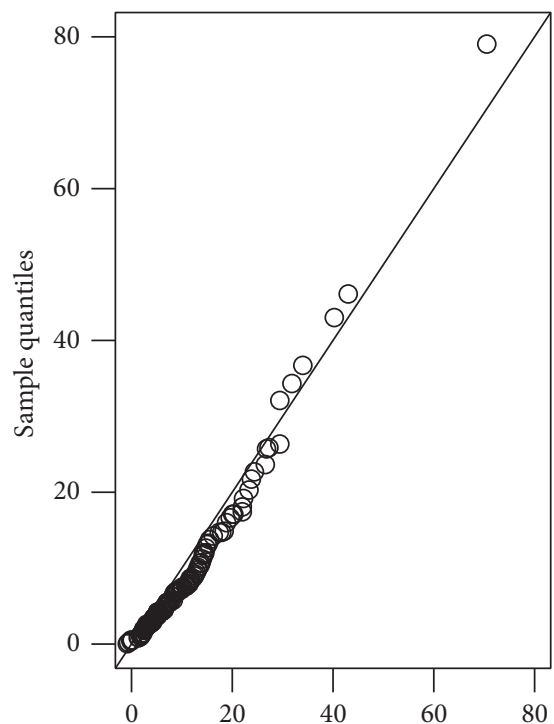

(e) Theoretical quantiles

FIGURE 3: Boxplot and qqplots for the remission times data set: PL (a), LP (b), SHN (c), SL (d), and MSL (e).

TABLE 2: Descriptive statistics for data set.

\begin{tabular}{lcccc}
\hline$n$ & $\bar{y}$ & $S$ & Asymmetry & Kurtosis \\
\hline 128 & 9.366 & 10.508 & 3.248 & 18.195 \\
\hline
\end{tabular}

$16.62,43.01,1.19,2.75,4.26,5.41,7.63,17.12,46.12,1.26,2.83$ $4.33,5.49,7.66,11.25,17.14,79.05,1.35,2.87,5.62,7.87,11.64$, $17.36,1.40,3.02,4.34,5.71,7.93,11.79,18.10,1.46,4.40,5.85$, $8.26,11.98,19.13,1.76,3.25,4.50,6.25,8.37,12.02,2.02,3.31$, 4.51, 6.54, 8.53, 12.03, 20.28, 2.02, 3.36, 6.76, 12.07, 21.73, 2.07, $3.36,6.93,8.65,12.63,22.69$. For this data set we present basic descriptive statistics and the maximum likelihood parameter estimates for the MSL model; see Tables 2 and 3. We compare these results with those of the SL, SHN, LP, and PL models, indicating the corresponding standard errors in parentheses.

Using the results from Section 3.1, moment estimators were computed leading to the following values (standard errors in parentheses): $\widehat{\lambda}_{M}=0.446(0.063)$ and $\widehat{q}_{M}=2.024$ (0.007); these were used as initial estimates for the maximum likelihood approach.

We calculated the Akaike information criterion AIC (see Akaike [13]) and the Bayesian information criterion BIC (see Schwarz [14]). These criteria reveal that the MSL model presents the best fit for the data set studied.

The left side of Figure 3 shows the boxplot of these data. The graphs of the QQ plots for the remission times data calculated with the MSL model fitted with the maximum 
TABLE 3: Summary statistics for a data set of remission times: model, ML estimates, and AIC and BIC values.

\begin{tabular}{lcrr}
\hline Model & ML estimates & AIC & BIC \\
\hline $\operatorname{MSL}(\lambda, q)$ & $\hat{\lambda}=0.374(0.020), \widehat{q}=2.803(0.032)$ & $\mathbf{8 2 3 . 8 3 5}$ & $\mathbf{8 2 9 . 5 5 9}$ \\
$\operatorname{SL}(\theta, \sigma, q)$ & $\widehat{\theta}=0.194(0.169), \widehat{\sigma}=0.535(0.534), \widehat{q}=2.100(0.512)$ & 825.445 & 834.002 \\
$\operatorname{SHN}(\sigma, q)$ & $\widehat{\sigma}=6.178(0.863), \hat{q}=2.038(0.447)$ & 826.287 & 831.991 \\
$\operatorname{LP}(\lambda, \theta)$ & $\hat{\lambda}=3.174(0.997), \widehat{\theta}=0.113(0.020)$ & 826.769 & 832.423 \\
$\operatorname{PL}(\alpha, \lambda)$ & $\widehat{\alpha}=0.830(0.047), \hat{\lambda}=0.294(0.037)$ & 830.708 & 836.411 \\
\hline
\end{tabular}

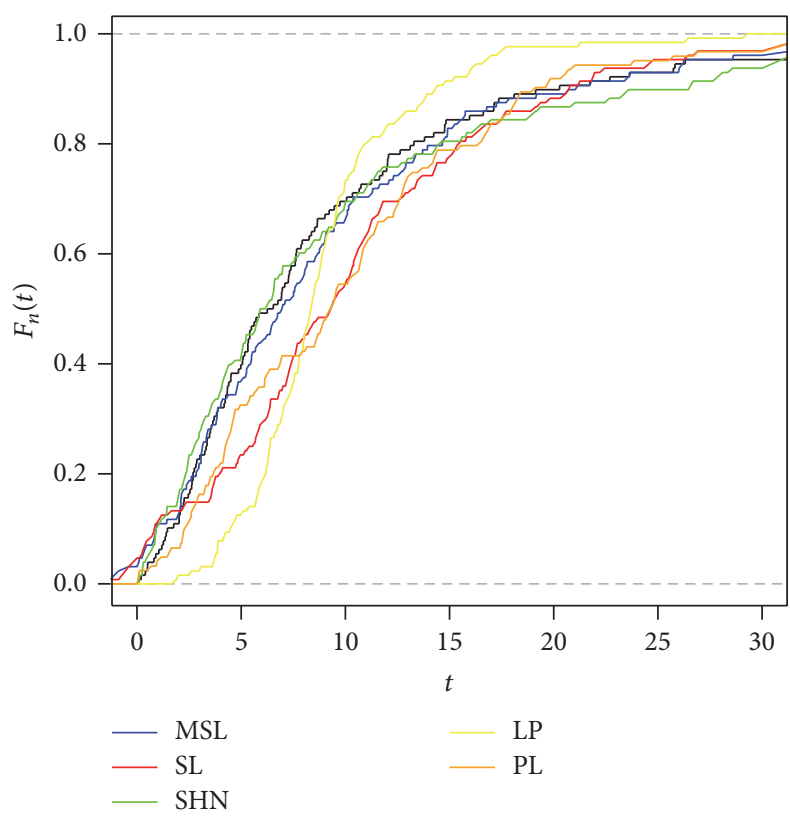

Figure 4: Models fitted by the maximum likelihood approach for the remission times data set: MSL (blue), SL (red), SHN (green), LP (yellow), and PL (orange).

likelihood estimates of the parameters; these are shown in Figure 3 compared with the SL, SHN, LP, and PL models. Figure 4 shows the empirical c.d.f. with the c.d.f. estimated by MSL, SL, SHN, LP, and PL; these also show the good agreement of the MSL model for the remission times data set.

\section{Discussion}

We introduce a new distribution with positive support based on the $L$ distribution, called Modified Slash Lindley distribution. It is generated as the quotient of two independent random variables, one being a Lindley distribution and the other a power of the exponential distribution. The Lindley distribution is a special case. Moments estimators are computed which can be used for initializing maximum likelihood estimation using Newton-Raphson. By computing the asymmetry and kurtosis coefficients we have illustrated that MSL is able to accommodate data with higher kurtosis. We present an illustration with a real data set where we show that the MSL distribution provides a better fit to data than the SL, SHN, LP, and PL distributions.

\section{Disclosure}

Preliminary results of this manuscript were presented as an abstract in a conference proceedings "XXV COMCA Congreso de Matemática Capricornio” 2016.

\section{Competing Interests}

The authors declare that there is no conflict of interests regarding the publication of this paper.

\section{Acknowledgments}

The research of J. Reyes and H. W. Gómez was supported by SEMILLERO UA-2016 (Chile).

\section{References}

[1] D. V. Lindley, "Fiducial distributions and Bayes' theorem," Journal of the Royal Statistical Society. Series B. Methodological, vol. 20, pp. 102-107, 1958.

[2] M. E. Ghitany, B. Atieh, and S. Nadarajah, "Lindley distribution and its application," Mathematics and Computers in Simulation, vol. 78, no. 4, pp. 493-506, 2008.

[3] P. Jodrá, "Computer generation of random variables with Lindley or Poisson-Lindley distribution via the Lambert $W$ function," Mathematics and Computers in Simulation, vol. 81, no. 4, pp. 851-859, 2010.

[4] M. E. Ghitany, D. K. Al-Mutairi, N. Balakrishnan, and L. J. AlEnezi, "Power Lindley distribution and associated inference," Computational Statistics and Data Analysis, vol. 64, pp. 20-33, 2013.

[5] N. M. Olmos, H. Varela, H. W. Gómez, and H. Bolfarine, "An extension of the half-normal distribution," Statistical Papers, vol. 53, no. 4, pp. 875-886, 2012.

[6] J. Reyes, H. W. Gómez, and H. Bolfarine, "Modified slash distribution," Statistics, vol. 47, no. 5, pp. 929-941, 2013.

[7] J. Reyes, H. W. Gómez, and I. Vidal, "Modified skew-slash distribution," Communications in Statistics. Theory and Methods, vol. 45, no. 4, pp. 1070-1080, 2016.

[8] J. Reyes, F. Vilca, D. I. Gallardo, and H. W. Gomez, "Modified slash Birnbaum-Saunders distribution," Hacettepe Journal of Mathematics and Statistics, vol. 46, no. 112, pp. 1-1, 2016.

[9] W. Gui, "Statistical properties and applications of the Lindley slash distribution," Journal of Applied Statistical Science, vol. 20, no. 3, pp. 283-298, 2012.

[10] W. Gui, S. Zhang, and X. Lu, “The Lindley-Poisson distribution in lifetime analysis and its properties," Hacettepe Journal of Mathematics and Statistics, vol. 43, no. 6, pp. 1063-1077, 2014. 
[11] P. K. Sen and J. M. Singer, Large Sample Methods in Statistics, Chapman \& Hall, New York, NY, USA, 1993.

[12] E. T. Lee and J. W. Wang, Statistical methods for survival data analysis, Wiley Series in Probability and Statistics, WileyInterscience [John Wiley \& Sons], Hoboken, NJ, USA, 3rd edition, 2003.

[13] H. Akaike, "A new look at the statistical model identification," IEEE Transactions on Automatic Control, vol. 19, no. 6, pp. 716$723,1974$.

[14] G. Schwarz, "Estimating the dimension of a model," The Annals of Statistics, vol. 6, no. 2, pp. 461-464, 1978. 


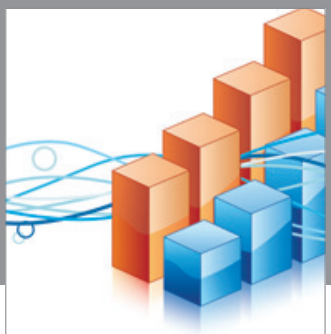

Advances in

Operations Research

vatem alat4

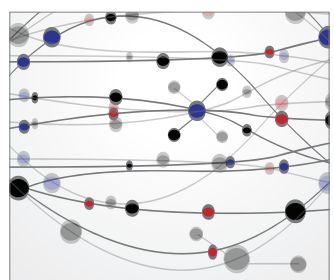

\section{The Scientific} World Journal
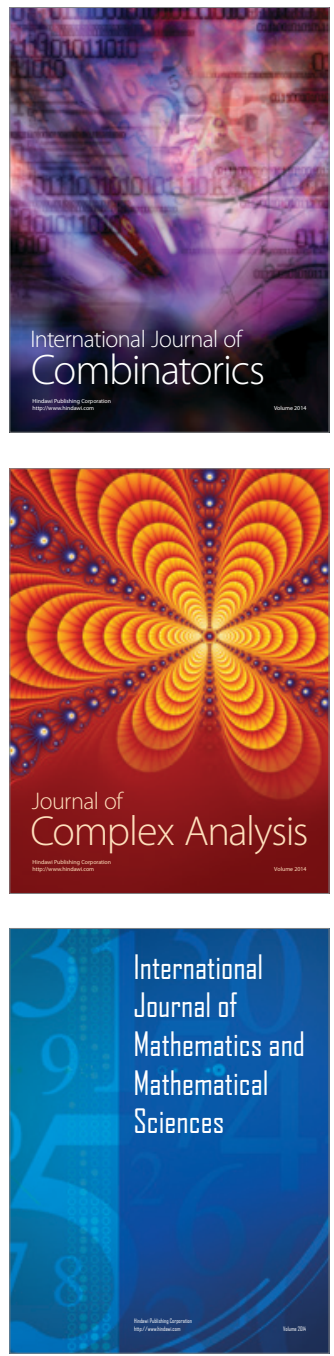
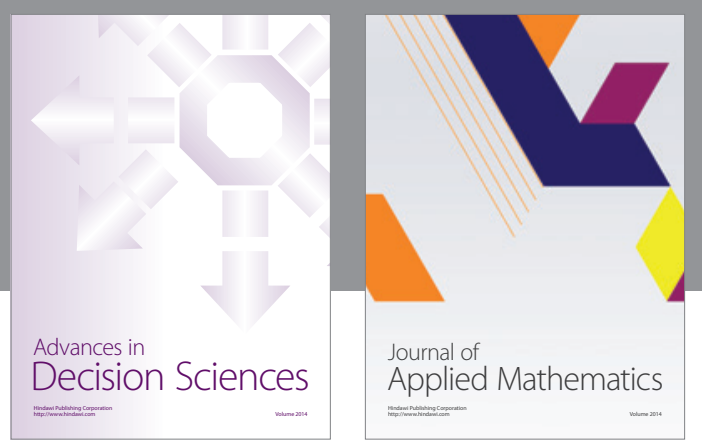

Algebra

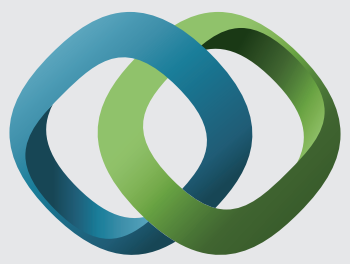

\section{Hindawi}

Submit your manuscripts at

https://www.hindawi.com
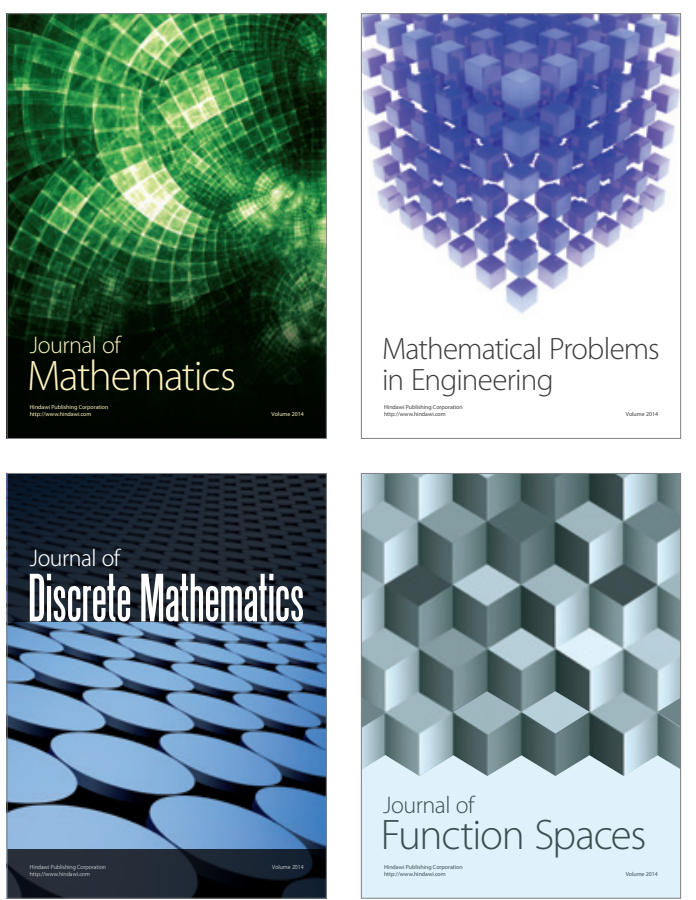

Mathematical Problems in Engineering
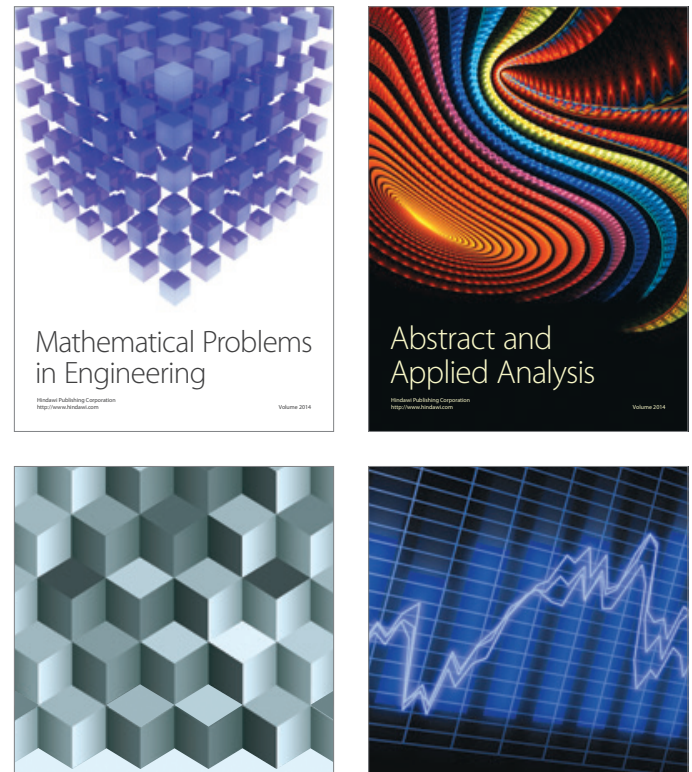

Journal of

Function Spaces

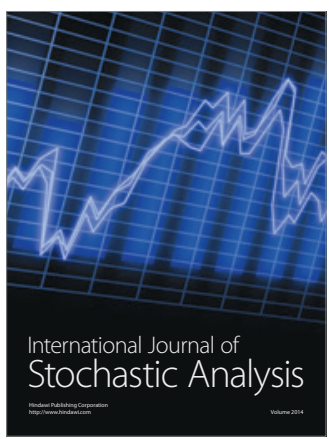

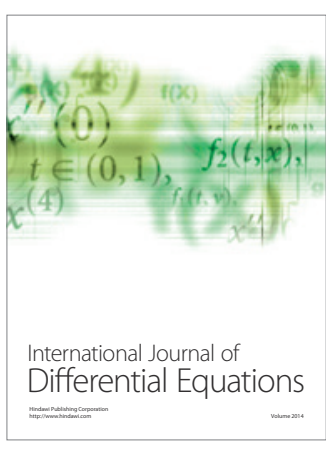
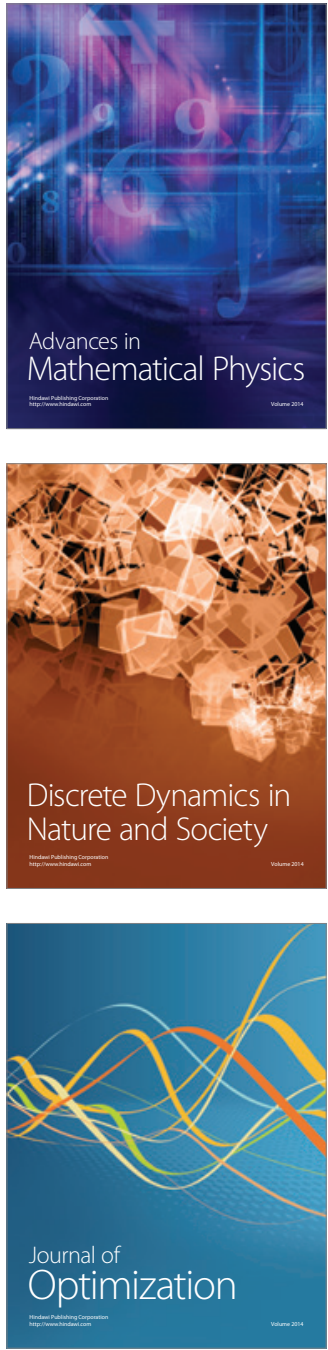